\title{
Changes in Electrophysiological Properties of Rat Skin with Age
}

\author{
Tanasait Ngawhirunpat, ${ }^{a}$ Tomomi Hatanaka, ${ }^{b}$ Kazunori Katayama, ${ }^{b}$ Hiroshi Yoshikawa, ${ }^{c}$ \\ Junichi KAWAKAMI, ${ }^{*}, a$ and Isao ADACHI ${ }^{a}$ \\ ${ }^{a}$ Department of Hospital Pharmacy, Toyama Medical and Pharmaceutical University; ${ }^{b}$ Department of Pharmaceutics, \\ Toyama Medical and Pharmaceutical University; ${ }^{c}$ Department of Drug Dosage Form Design, Faculty of Pharmaceutical \\ Sciences, Toyama Medical and Pharmaceutical University; 2630 Sugitani, Toyama 930-0194, Japan.
}

Received January 22, 2002; accepted May 31, 2002

The age-related changes in the electrical and physiological properties of the skin were examined in rats at the ages of 5, 10, 21, 90, and $180 \mathrm{~d}$. The resistance of the stratum corneum, the resistance of the viable skin (epidermis and dermis), and the capacitance of the stratum corneum were analyzed from skin impedance data using an equivalent circuit. With development and aging, the resistance of the stratum corneum and the viable skin increased, whereas the capacitance of the stratum corneum decreased. Physiological characteristics such as the thickness of skin strata and the content of lipid and water in the stratum corneum were also measured. The lipid content in the stratum corneum was constant at all ages. The water content in the stratum corneum decreased, and the thickness of skin strata increased with age. Comparison between electrical data and physiological properties suggested that the increase in the resistance of the stratum corneum with aging is primarily caused by the decrease in the water content and that the capacitance of the stratum corneum and the resistance of the viable skin depend on age-related increases in the thickness of skin strata. In conclusion, the age dependency of cutaneous electrical properties may affect the permeation profile of drugs through the skin, and impedance analysis can be used to estimate age-related changes in transdermal drug delivery.

Key words development; aging; electrical impedance analysis; skin absorption

Skin age is one of the biological factors affecting percutaneous absorption. ${ }^{1,2)}$ To regulate the therapeutic efficacy of transdermal drug delivery, the difference in skin permeability at various ages is a subject of considerable importance. We previously reported that the permeability through the skin decreases with aging, ${ }^{3,4)}$ and that the extent of this decrease is higher for lipophilic permeants than for hydrophilic permeants. ${ }^{4)}$ We clarified that the thickness of skin strata is an important factor for limited percutaneous absorption in aging. However, other physiological changes in the skin with age, e.g., moisture content in the skin, lipid composition, lipid content, dermis thickness, and density of skin appendages, have been also reported. ${ }^{5-8)}$ Therefore the age-related differences in the skin barrier function must be further investigated based on physiological alterations in the skin to elucidate the mechanisms of the age dependency of transdermal absorption of drugs.

There have been many reports on age-related changes in the transdermal permeation of drugs, ${ }^{3,4,6-9)}$ however, age-related changes in skin permeability have not been fully interpreted because of large interindividual variance and the limited number of model permeants. Recently, it was reported that impedance parameters can sensitively reflect the properties of skin permeation pathways. ${ }^{10-12)}$ The impedance technique enables us to determine rapidly changes in skin physical properties, and one measurement provides information on pathways for both hydrophilic and lipophilic permeants. Therefore skin impedance analysis may be a useful method to elucidate the skin barrier function at different ages.

In the present study, we attempted to apply impedance analysis to investigate age-dependent changes in permeation pathways of rat skin. In vitro skin impedance data of rats at various ages were compared. Physiological characteristics of the skin, e.g., the water and lipid content of the stratum corneum and the thickness of the skin strata, were also evalu- ated. Finally, we demonstrated the age dependency of relations between these electrical and physiological properties.

\section{MATERIALS AND METHODS}

Animals Male STD: Wistar rats at the age of 5, 10, 21, 90, and $180 \mathrm{~d}$ (Japan SLC Inc., Hamamatsu, Japan) were used. Rats were fed commercial food pellets and tap water $a d$ libitum. Under sodium pentobarbital anesthesia $(50 \mathrm{mg} / \mathrm{kg}$, i.p.), a round section of the abdominal skin was excised, and then the subcutaneous fat and other extraneous tissue were trimmed off carefully. The skin sample was immediately used in the following skin impedance experiments.

Skin Impedance Measurement and Analysis A sideby-side, two-chamber electrochemical cell with four electrodes, two for supplying current and two for recording signals, was designed according to the procedure of Burnett and Bagniefski. ${ }^{11)}$ The rat skin was mounted in an O-ring seal between the two chambers, each having $23 \mathrm{ml}$ volume and $1 \mathrm{~cm}^{2}$ exposed area. The current-supplying electrode consisted of $0.79 \mathrm{~cm}^{2}$ of a platinum black plate which was fixed at each edge of the chamber and positioned $6 \mathrm{~cm}$ from the skin membrane according to the method of Hanai. ${ }^{13)}$ The measuring electrode was $15 \mathrm{~mm}$ of silver-silver chloride electrodes fabricated by the method of Janz and Ives ${ }^{14)}$ and placed at a distance of $7 \mathrm{~mm}$ from the membrane surface.

The electrochemical cell was immersed in a water bath at $37^{\circ} \mathrm{C}$ throughout the impedance measurements. The two chambers were filled with $0.9 \%$ sodium chloride isotonic solution, and then stirred at $1440 \mathrm{rpm}$ with a magnetic starhead bar driven by a synchronous motor for $14 \mathrm{~h}$ to obtain full hydration of skin samples. Saline in both chambers was replaced with fresh solution. A sinusoidal current, produced by a signal generator (Model 33120, Hewlett Packard, Corvallis, OR, U.S.A.), was applied via a $100-\mathrm{k} \Omega$ resistor at an 
amplitude of $10 \mathrm{~V}$ to the current-supplying electrodes in the electrochemical cell. The current was determined primarily by the $100-\mathrm{k} \Omega$ resistor and thus almost constant $\left(0.12 \mathrm{~mA} / \mathrm{cm}^{2}\right.$ of skin) for all measurements. The potential difference across the skin was determined by measuring the voltage at each measuring electrode using a digital oscilloscope (Model 310, Nicolet, Madison, WI, U.S.A.). The determination was carried out over the frequency range of 10 $200000 \mathrm{~Hz}$, with three or four frequency points per logarithmic decade. Four thousand data points/each measurement collected on the oscilloscope at a sampling rate of 2$1000 \mathrm{kHz}$ were transferred to a personal computer (PC-9801, NEC, Tokyo, Japan) for storage and subsequent analysis. The collected data were fitted to a sinusoidal function using a nonlinear least-squares method based on Berman et al.'s algorithm, ${ }^{15)}$ and the amplitude was estimated. The amplitude was converted to the skin impedance according to Ohm's law based on the constant current.

An equivalent circuit consisting of a parallel combination of resistance $\left(R_{\mathrm{S} 1}\right)$ and capacitance $\left(C_{\mathrm{S}}\right)$, which is a model of the stratum corneum, in series with the resistance $\left(R_{\mathrm{S} 2}\right)$ corresponding to the viable skin (the epidermis and dermis), as shown in Fig. 1a, was used in the impedance analysis as reported in previous studies. ${ }^{16,17)}$ Based on the circuit, the impedance $(|Z|)$ is given by:

$$
|Z|=\sqrt{\frac{\left(R_{\mathrm{S} 1}+R_{\mathrm{S} 2}\right)^{2}+\left(R_{\mathrm{S} 1} R_{\mathrm{S} 2} C_{\mathrm{S}} \omega\right)^{2}}{1+\left(R_{\mathrm{S} 1} C_{\mathrm{S}} \omega\right)^{2}}}
$$

where $\omega$ is the angular frequency $(\omega=2 \pi f)$ and $f$ is the frequency. The impedance data over the frequency range of $10-200000 \mathrm{~Hz}$ were simultaneously fitted to Eq. 1 by nonlinear least-squares regression to estimate the values of $R_{\mathrm{S} 1}$, $R_{\mathrm{S} 2}$, and $C_{\mathrm{S}} \cdot{ }^{15)}$

Measurement of Skin Thickness The thickness of the stratum corneum, viable epidermis, dermis, and whole skin of rats was determined according to the method of Evans and Rutter with a minor modification. ${ }^{18)}$ The skin specimen was fixed in $3 \%$ formaldehyde in $0.1 \mathrm{~m}$ phosphate buffer $(\mathrm{pH} 7.2)$ and was dipped in paraffin. The sections were cut and stained with hematoxylin-eosin, and the thickness of the stratum corneum $\left(L_{\mathrm{SC}}\right)$ and the viable skin $\left(L_{\mathrm{VS}}\right)$, composed of viable epidermis and dermis, were then observed under light microscopy (BHS-324, Olympus, Tokyo).

Measurement of Lipid Content in the Stratum Corneum The stratum corneum of the rat skin was isolated by trypsin treatment. ${ }^{19)}$ The dermis side of the whole skin was placed on a filter paper saturated with $1 \% \mathrm{w} / \mathrm{v}$ trypsin solution (10000 ATEE units $\mathrm{ml}^{-1}$ in phosphate-buffered saline at $\mathrm{pH} 7.4$ at $37^{\circ} \mathrm{C}$ in a sealed Petri dish for $8 \mathrm{~h}$. After this incubation, the stratum corneum was carefully separated from the viable epidermis and rinsed thoroughly with distilled water.

The content of lipid and total lipid, and lipid composition of ceramides, cholesterol, and phospholipids in the stratum corneum, were determined. After weighing, samples of the dry stratum corneum (about $10 \mathrm{mg}$ ) were placed in a $10-\mathrm{ml}$ glass tube with a screw cap containing $7 \mathrm{ml}$ of $2: 1$ chloroform/methanol mixture and shaken for $20 \mathrm{~h}$ at room temperature. The delipidized stratum corneum samples were transferred to another tube, rinsed with fresh chloroform/methanol mixture, and dried to constant weight. The total lipid content of the stratum corneum was determined from the difference in its weight before and after solvent extraction. The lipid composition in the extracted solvent was also analyzed. Ceramide content was determined according to the method of Lauter and Trams. ${ }^{20}$ Cholesterol and phospholipids were measured using the cholesterol CII-testWako ${ }^{\circledR}$ and phospholipid B-testWako ${ }^{\circledR}$ (Wako Pure Chemical Industries, Osaka, Japan). The lipid content was calculated in units of milligrams of the lipid per milligram of the stratum corneum.

Measurement of Water Content in the Stratum Corneum The measurement of water content in the stratum corneum was performed according to the method of Potts et al. ${ }^{21)}$ Briefly, the water content in the skin was quantified by measuring the intensity of the weak $\mathrm{O}-\mathrm{H}$ stretch near $2100 \mathrm{~cm}^{-1}$ in the infrared spectra. The stratum corneum was isolated by the same method as for lipid content measurement. The stratum corneum was analyzed using Fourier transform infared spectroscopy with an ATR sampling device (FT/IR-230, JASCO, Tokyo, Japan). All in vitro spectra were obtained under ambient laboratory conditions of approximately $40 \%$ relative humidity at $25^{\circ} \mathrm{C}$. The water content in the skin was calculated as the ratio of mass of water to dry mass of the stratum corneum (mg/mg).

Statistical Analyses Analysis of variance (ANOVA) with Dunnet's test in multiple comparison was used for statistical evaluation of the thickness of skin strata, lipid and water content in stratum corneum, skin impedance, and relation between skin impedance with thickness at each age, respectively. $p$ values of $<0.05$ were considered to represent a statistically significant difference.

\section{RESULTS}

Figure $1 \mathrm{~b}$ shows a typical logarithmic plot of $|Z|$ against $f$ of the excised skin of rats at the age of $90 \mathrm{~d}$. Three spectral regions, a horizontal impedance response at $10-1000 \mathrm{~Hz}$, a rapid decline at $1000-80000 \mathrm{~Hz}$, and an extrapolated horizontal line above $80000 \mathrm{~Hz}$, were observed. The solid curve in Fig. 2 indicates the best-fitted data to Eq. 1. The estimated values (mean \pm S.D.) of $R_{\mathrm{S} 1}, C_{\mathrm{S}}$, and $R_{\mathrm{S} 2}$ were $1433 \pm 56$ $\Omega \cdot \mathrm{cm}^{2}, 33 \pm 4 \mathrm{nF} / \mathrm{cm}^{2}$, and $80 \pm 2 \Omega \cdot \mathrm{cm}^{2}$, respectively. $R_{\mathrm{S} 1}$, $R_{\mathrm{S} 2}$, and $C_{\mathrm{S}}$ at other ages were measured and are plotted against age in Figs. $2 \mathrm{a}-\mathrm{c}$, respectively. $R_{\mathrm{S} 1}$ and $R_{\mathrm{S} 2}$ increased markedly from $5 \mathrm{~d}$ to $90 \mathrm{~d}$ and then gradually to $180 \mathrm{~d}$. The $R_{\mathrm{S} 1}$ value was much higher than $R_{\mathrm{S} 2}$ at each age. On the other hand, $C_{\mathrm{S}}$ decreased markedly from 5 to $90 \mathrm{~d}$ and then gradually to $180 \mathrm{~d}$.

Table 1 shows the thickness of the stratum corneum, viable epidermis, dermis, and whole skin of rats at various ages. $L_{\mathrm{SC}}$ gradually increased with age. $L_{\mathrm{VS}}$ increased with age, and in particular the thickness of the dermis dramatically increased from 21 to $90 \mathrm{~d}$. To exclude the effect of the thickness of skin strata on the skin impedance parameters, the relation between age and $R_{\mathrm{S} 1} / L_{\mathrm{SC}}, R_{\mathrm{S} 2} / L_{\mathrm{VS}}$ and the product of $C_{\mathrm{S}}$ and $L_{\mathrm{SC}}$ $\left(C_{\mathrm{S}} \cdot L_{\mathrm{SC}}\right)$ were determined and are illustrated in Figs. $3 \mathrm{a}-\mathrm{c}$, respectively. With aging, the ratio of $R_{\mathrm{S} 1} / L_{\mathrm{SC}}$ increased, whereas $R_{\mathrm{S} 2} / L_{\mathrm{VS}}$ and $C_{\mathrm{S}} \cdot L_{\mathrm{SC}}$ remained almost constant.

Table 2 shows the lipid and water content in the stratum corneum of rats at various ages. The lipid composition and total lipid in the stratum corneum did not differ significantly 
(a)

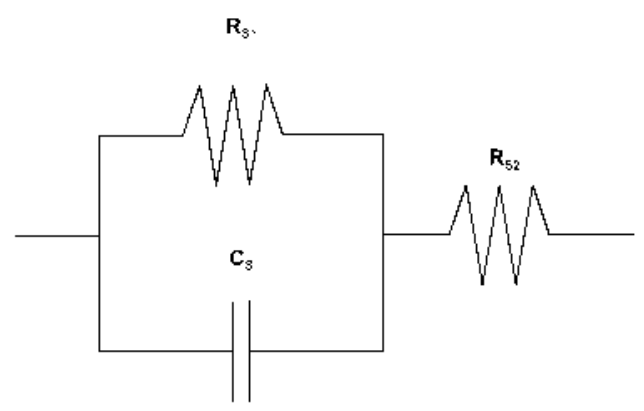

(b)

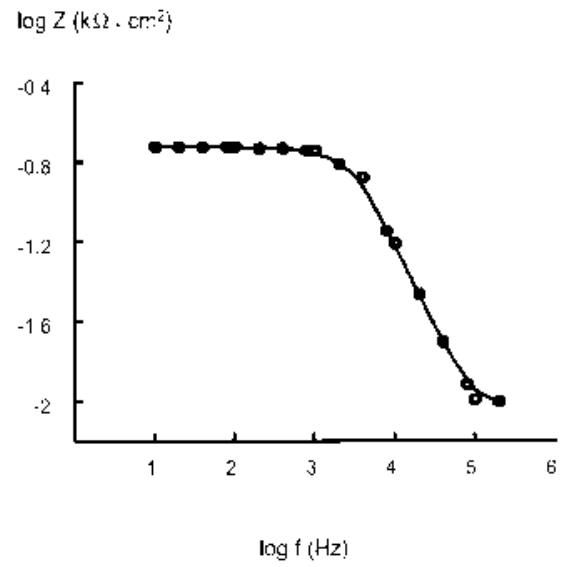

Fig. 1. Equivalent Circuit of Skin (a) and Logarithmic Plot of Impedance against Frequency of Excised Skin of Rats at the Age of $90 \mathrm{~d}$ (b)

In panel (a), $R_{\mathrm{S} 1}$ is the resistance of the stratum corneum, $C_{\mathrm{S}}$ is the capacitance of the stratum corneum, and $R_{\mathrm{S} 2}$ is the resistance of the viable epidermis and dermis.

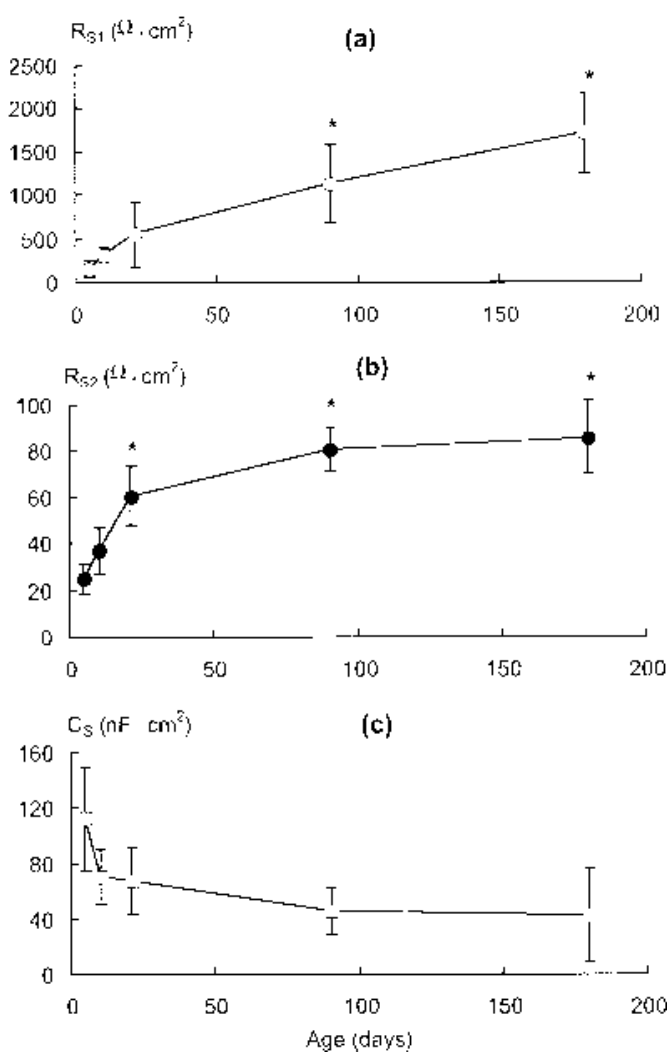

Fig. 2. Age Dependency of $R_{\mathrm{S} 1}(\mathrm{a}), R_{\mathrm{S} 2}(\mathrm{~b})$, and $C_{\mathrm{S}}(\mathrm{c})$ in Rat Skin Each point represents the mean \pm S.D. of three to four experiments. $* p<0.05$ compared with rats at $5 \mathrm{~d}$

Table 1. Thickness of the Skin Strata of Rats at Various Ages ${ }^{a)}$

\begin{tabular}{rccccc}
\hline \hline Age $(\mathrm{d})$ & Stratum corneum $\left(L_{\mathrm{SC}}\right)(\mu \mathrm{m})$ & Epidermis $(\mu \mathrm{m})$ & Dermis $(\mu \mathrm{m})$ & Epidermis + Dermis $\left(L_{\mathrm{VS}}\right)(\mu \mathrm{m})$ & Whole skin $(\mathrm{mm})$ \\
\hline 5 & $13.9 \pm 3.2$ & $13.8 \pm 2.2$ & $243.3 \pm 29.8$ & $267.1 \pm 31.1$ & $0.276 \pm 0.039$ \\
10 & $16.5 \pm 2.4$ & $14.8 \pm 2.5$ & $311.3 \pm 31.4$ & $326.2 \pm 34.0$ & $0.330 \pm 0.046$ \\
21 & $18.1 \pm 2.7$ & $15.9 \pm 2.5$ & $350.6 \pm 42.8$ & $366.5 \pm 43.3$ & $0.369 \pm 0.044$ \\
90 & $23.4 \pm 2.9^{*}$ & $18.9 \pm 2.4$ & $720.8 \pm 51.9^{*}$ & $739.7 \pm 54.3^{*}$ & $0.777 \pm 0.052^{*}$ \\
180 & $27.5 \pm 3.8^{*}$ & $22.7 \pm 3.4^{*}$ & $808.8 \pm 69.3^{*}$ & $831.5 \pm 72.8^{*}$ & $0.853 \pm 0.077^{*}$ \\
\hline
\end{tabular}

a) Each value represents the mean \pm S.D. of three to four experiments. $* p<0.05$ compared with rats at $5 \mathrm{~d}$.

among rats of different ages. On the other hand, the water content in the stratum corneum decreased markedly from 5 to $21 \mathrm{~d}$ and then gradually from 21 to $180 \mathrm{~d}$.

\section{DISCUSSION}

In the present study, the alteration of the electrical response of the skin with aging was evaluated using impedance analysis using an equivalent circuit, which was a parallel combination of $R_{\mathrm{S} 1}$ and $C_{\mathrm{S}}$, in series with another resistance, $R_{\mathrm{S} 2}$ (Fig. 1a). This circuit is the most widely used as a simple model of an equivalent circuit of the skin. ${ }^{22} R_{\mathrm{S} 1}$, the main barrier of the skin, was markedly higher than $R_{\mathrm{S} 2}$ at each age (Figs. 2a, b). The $R_{\mathrm{S} 2}$ value was consistent with the data obtained in the stripped skin in our preliminary experiment. The values of electrical parameters in this study were within the range of those in previous reports of the human and hairless mouse skin. ${ }^{10-12)}$ Our results supported the validity of the use of the equivalent circuit in studies of this type.

$R_{\mathrm{S} 1}$ increased with age (Fig. $2 \mathrm{a}$ ). $R_{\mathrm{S} 1}$ reflects the properties of pathways for hydrophilic permeants or pore pathways in skin permeation. ${ }^{11,12,23)}$ Skin permeability of diclofenac sodium (DC-Na) and deuterium oxide $\left(\mathrm{D}_{2} \mathrm{O}\right)$ were reported to decrease with age. ${ }^{3)}$ To elucidate the factors involved in age-related changes in $R_{\mathrm{S} 1}$, thickness and water content were measured. An increase in $L_{\mathrm{SC}}$ with age was observed (Table 1 ). However, the tendency of $R_{\mathrm{Sl}} / L_{\mathrm{SC}}$ to increase with age (Fig. 3a) means that the thickness may not be the only factor contributing to age-related differences in $R_{\mathrm{S} 1}$, and the volume of pore pathways or porosity of the skin may decrease with increasing age. A decrease in water content of the stratum corneum with aging was found (Table 2). Similar data have been reported by other investigators. ${ }^{24)}$ Differential scanning calorimetric studies suggested that the cutaneous water promotes the fluidity of the lipid bilayer and extends the lipid domain among the lipid polar groups. ${ }^{25)}$ As the stratum 
corneum becomes more hydrated, the ion in the stratum corneum can move more freely and is more responsive to an applied electric field. Water content in the stratum corneum may play an important role in the age-related increase in $R_{\mathrm{S} 1}$. In our previous permeation study, the effect of water content on the skin permeation of DC-Na and $\mathrm{D}_{2} \mathrm{O}$ could not be detected, because its effect might be masked by large interindividual variance of permeation data. Further permeation studies using other hydrophilic permeants are required to confirm this effect of water content.

$R_{\mathrm{S} 2}$ also increased with aging (Fig. 2b). Increased $L_{\mathrm{VS}}$ with aging (Table 1) and constant values of $R_{\mathrm{S} 2} / L_{\mathrm{VS}}$ at all ages (Fig. 3b) indicate that $L_{\mathrm{VS}}$ is a major factor in the age dependency in $R_{\mathrm{S} 2}$. Because $R_{\mathrm{S} 2}$ were markedly lower than $R_{\mathrm{S} 1}$ at all ages, the age-related changes may not affect permeation profiles through the skin, except in stripped skin, as reported previously. ${ }^{3)}$

In contrast, $C_{\mathrm{S}}$ has a tendency to decrease with aging (Fig.
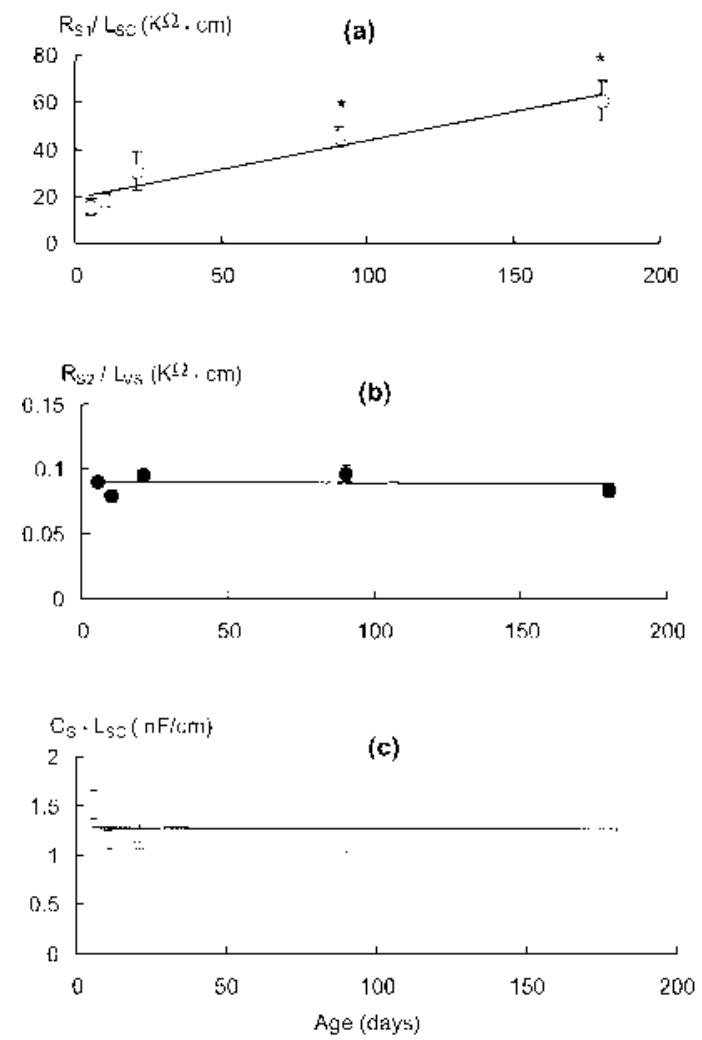

Fig. 3. Age Dependency of $R_{\mathrm{S} 1} / L_{\mathrm{SC}}(\mathrm{a}), R_{\mathrm{S} 2} / L_{\mathrm{VS}}$ (b), and the Product of $C_{\mathrm{S}}$ and $L_{\mathrm{SC}}(\mathrm{c})$

Each point represents the mean \pm S.D. of three to four experiments. A linear regression line was drawn in each graph. $* p<0.05$ compared with rats at $5 \mathrm{~d}$. 2c). It has been reported that the capacitance is due to the insulating properties of the lipid-cellular matrix, ${ }^{22)}$ and thus reflects the lipid pathway, i.e., the skin permeation route for lipophilic permeants, in the stratum corneum. ${ }^{10-12)}$ The decreased $C_{\mathrm{S}}$ with aging represents the development of the skin barrier function against lipophilic permeants. We have already reported that the skin permeability of ketoprofen and isosorbide dinitrate is lower at older ages. ${ }^{3)}$ We thus focused on the two main factors of lipid content and $L_{\mathrm{SC}}$ in the agerelated alteration of capacitance. The content of cholesterol, phospholipids, and ceramides and total lipid in the stratum corneum was not significantly different at any age (Table 2 ). On the other hand, $L_{\mathrm{SC}}$ increased with age (Table 1), while $C_{\mathrm{S}} \cdot L_{\mathrm{SC}}$ remained almost constant at all ages (Fig. $3 \mathrm{c}$ ). These results strongly suggest that $L_{\mathrm{SC}}$ plays an important role in the age-related changes in $C_{\mathrm{S}}$ and skin permeation of lipophilic permeants.

In the present study, skin impedance analysis was performed to investigate age-related changes in the skin barrier function in rats. With aging, $R_{\mathrm{S} 1}$ and $R_{\mathrm{S} 2}$ increased, whereas $C_{\mathrm{S}}$ decreased. These findings suggest that skin permeability to both lipophilic and hydrophilic permeants may decrease with age. The discrepancy in $R_{\mathrm{S} 1}$ among ages was mainly attributed to the water content in the stratum corneum, whereas the variability in $R_{\mathrm{S} 2}$ and $C_{\mathrm{S}}$ was primarily due to the thickness of skin strata, as represented by $L_{\mathrm{SC}}$ and $L_{\mathrm{VS}}$, respectively. The influence of cutaneous water on $R_{\mathrm{S} 1}$ was a primary factor in the age-related changes in the skin barrier function, which was not found in our previous study of skin permeation. ${ }^{3)}$ In conclusion, impedance analysis may be a useful method to study age-related changes in transdermal absorption.

\section{REFERENCES}

1) Roskos K. V., Maibach H. I., Guy R. H., J. Pharmacokin. Biopharm., 17, 617-630 (1990).

2) Roskos K. V., Maibach H. I., Drugs Aging, 2, 432-449 (1992).

3) Ngawhirunpat T., Yoshikawa H., Hatanaka T., Koizumi T., Adachi I., Pharmazie, 56, 231-234 (2001).

4) Ngawhirunpat T., Hatanaka T., Kawakami J., Adachi I., Biol. Pharm. Bull., 24, 414 - 417 (2001).

5) Andrew W., Bennke R. H., Sato T., Gerontologia, 10, 1-19 (1965).

6) Shuster S., Black M. M., McVite E., Br. J. Dermatol., 93, 639-643 (1975).

7) Knaak J. B., Yee K., Ackerman C. R., Zweig G., Wilson B. W., Toxicol. Appl. Pharmacol., 72, 406-416(1984).

8) Dick I. P., Scott R. C., Pharm. Res., 9, 884-887 (1992).

9) Fisher H. L., Shah P. V., Sumler M. R., Hall L. L., Environ. Res., 50, 120-139 (1989).

10) Yamamoto T., Yamamoto Y., Med. Biol. Eng., 14, 151-158 (1976).

11) Burnett R. R., Bagniefski T. M., J. Pharm. Sci., 77, 492- 497 (1988).

Table 2. Lipid and Water Content in the Stratum Corneum of Rats at Various Ages ${ }^{a)}$

\begin{tabular}{|c|c|c|c|c|c|c|}
\hline \multirow{2}{*}{ Content } & \multirow{2}{*}{ Composition } & \multicolumn{5}{|c|}{ Age (d) } \\
\hline & & 5 & 10 & 21 & 90 & 180 \\
\hline \multirow{4}{*}{ Lipid $(\mu \mathrm{g} / \mathrm{mg})$} & Ceramides & $66.3 \pm 7.9$ & $68.5 \pm 7.1$ & $65.8 \pm 9.6$ & $61.8 \pm 8.2$ & $63.4 \pm 7.1$ \\
\hline & Cholesterol & $37.7 \pm 8.1$ & $38.5 \pm 9.6$ & $37.4 \pm 6.5$ & $33.9 \pm 8.6$ & $35.5 \pm 5.3$ \\
\hline & Phospholipids & $0.41 \pm 0.08$ & $0.38 \pm 0.06$ & $0.35 \pm 0.05$ & $0.36 \pm 0.04$ & $0.32 \pm 0.06$ \\
\hline & Total extracted lipid & $150 \pm 15.2$ & $144 \pm 7.5$ & $150 \pm 7.8$ & $145 \pm 10.5$ & $147 \pm 12.2$ \\
\hline Water $(\mathrm{mg} / \mathrm{mg})$ & & $0.12 \pm 0.02$ & $0.071 \pm 0.006$ & $0.062 \pm 0.005^{*}$ & $0.055 \pm 0.005^{*}$ & $0.051 \pm 0.004^{*}$ \\
\hline
\end{tabular}

a) Each value represents the mean \pm S.D. of four to five experiments. $* p<0.05$ compared with rats at $5 \mathrm{~d}$. 
12) Inada H., Ghanem A. H., Higuchi W. I., Pharm. Res., 11, 687-697 (1994).

13) Hanai T. (ed.), "Membrane and Ions: Principles and Techniques of Mass Transport," Kagaku Doujin, Tokyo, 1978, pp. 1-25.

14) Janz G., Ives D., Ann. N.Y. Acad. Sci., 148, 210-220 (1968).

15) Berman M., Shan E., Weiss M. F., Biophys. J., 2, 275-282 (1962).

16) DeNuzzio J. D., Berner B., J. Control. Rel., 69, 105-112 (1990).

17) Woolfson A. D., Moss G. P., McCafferty D. F., Lackermeier A., McAdams E. T., Pharm. Res., 16, 459-462 (1999).

18) Evans N. J., Rutter N., Biol. Neonate, 49, 74-80 (1986).

19) Knutson K., Potts R. O., Guzek D. B., Golden G. M., Mckie J. E.,
Lambert W. J., Higuchi W. I., J. Control. Rel., 2, 67-87 (1985).

20) Lauter C. J., Trams E. G., J. Lipid. Res., 3, 136-148 (1962).

21) Potts R. O., Guzek D. B., Harris R. R., Mckie J. E., Arch. Dermatol. Res., 277, 489-495 (1985).

22) Nolan I. M. A., Corish J., Corrigan O. I., Chem. Soc. Faraday Trans., 89, 2839-2845 (1993).

23) Potts R. O., Guy R. H. (eds.), "Mechanisms of Transdermal Drug Delivery," Marcel Dekker, New York, 1997, pp. 267-290.

24) Potts R. O., Buras E. M., Chrisman D. A., J. Invest. Dermatol., 82, 97-100 (1984).

25) Barry B. W., J. Control. Rel., 6, 85-97 (1987). 